\title{
CONSUMO E EXCREÇÃO DE CÁLCIO EM IDOSOS INSTITUCIONALIZADOS DO CONCELHO DE BRAGANÇA
}

\author{
CALCIUM CONSUMPTION AND EXCRETION IN INSTITUTIONALIZED ELDERLY FROM \\ BRAGANÇA REGION, PORTUGAL \\ INGESTIÓN Y EXCRECIÓN URINNARIA DE CALCIO EN ANCIANOS \\ INTITUCIONALIZADOS EN EL MUNICIPIO DE BRAGANÇA, PORTUGAL
}

\author{
Ana Teresa Domingues Gonçalves* \\ Andreia Raquel Fernandes de Oliveira* \\ António José Andrade Pereira* \\ Jerusa Vieira Delindro* \\ António José Gonçalves Fernandes** \\ Juliana Almeida-de-Souza**
}

\section{RESUMO:}

Este estudo pretendeu verificar a relação da ingestão de cálcio, sódio e proteína com a excreção urinária de cálcio, bem como investigar se idosos com patologias apresentam diferenças na calciúria. Para isso, foi realizado um estudo quantitativo, observacional, transversal e analítico com base numa amostra inicial de residentes em lares do concelho de Bragança. Recolheram-se os dados pessoais, antropométricos bem como informação acerca da existência de patologias. Para estimar o consumo de micro e macronutrientes foi aplicado um recordatório de 24 horas e para a avaliação da calciúria foram recolhidas amostras da urina de 24 horas. O tratamento dos dados envolveu o uso do SPSS 21.0 e dos testes de Wilcoxon, Spearman e Mann-Whitney-Wilcoxon. Os resultados demonstraram que a maioria dos idosos consome sódio e proteína acima do recomendado e cálcio abaixo do recomendado. As patologias não se mostraram relacionadas com a calciúria. Verificou-se, igualmente, que o consumo e a excreção de cálcio não estavam correlacionados. O estudo da associação entre a calciúria e o consumo de proteína e sódio revelou a existência de correlações positivas, embora fracas. Conclui-se que, para além de controlar a ingestão de cálcio em idosos institucionalizados, torna-se necessário adequar o consumo de sódio e proteína aos valores recomendados, de forma a minimizar as perdas de cálcio na urina.

Palavras-chave: Ingestão, Excreção, Cálcio, Idosos, Bragança.

\footnotetext{
ABSTRACT:

This study aims to verify whether there is a relationship between the calcium, sodium and protein intake and the calcium urinary excretion, as well as to investigate whether elderly patients with pathologies
} 


\section{Egitania \\ s c i e $\cap$ c i a}

show urine calcium differences. A quantitative, observational, cross-sectional analytical study was carried out based on a sample of residents in nursing homes in the town of Bragança, Portugal. Personal and anthropometric data and information about the existence of pathologies were collected. For the consumption of micro and macronutrients a 24 hour recall was applied and for evaluation of urinary calcium 24 hour urine was collected. Data treatment involved the use of SPSS 21.0 and Wilcoxon, Spearman and Mann-Whitney-Wilcoxon tests. The results of this study show that most elderly consume sodium and protein above the recommended intake and calcium below the recommended level. Pathologies and calcium excretion were not related. Also, it was found that the calcium intake and excretion were not correlated. The study of the association between calcium excretion and sodium and protein intake revealed a positive but weak correlation. It is concluded that, in addition to controlling the calcium intake in institutionalized elderly, it becomes necessary to adjust the amounts of sodium and protein intake to minimize the loss of calcium in the urine.

Key-words: Ingestion, Excretion, Calcium, Elderly, Bragança.

\section{RESUMEN:}

Este estudio tiene como objetivo verificar si existe una relación entre la ingestión de calcio, sodio y de proteínas con la excreción urinaria de cálcio e investigar si las patologías interfieren en la excreción de calcio de los pacientes de edad avanzada. En este estudio cuantitativo, observacional, transversal y analítico se obtuvo una muestra de institucionalizados en el municipio de Bragança, Portugal. Se recogieron datos personales, antropométricos y patológicos de los ancianos. Para estimar el consumo de micro y macro nutrientes se aplicó un recordatorio de 24 horas y para la evaluación de la calciuria se recogieron muestras de orina de 24 horas. El tratamiento de los datos implicó el uso de SPSS 21.0 y las pruebas de Wilcoxon, Spearman y Mann-Whitney-Wilcoxon. Los resultados demostraron que la mayoría de los ancianos consume sodio y proteínas por encima de las cantidades recomendadas y calcio por debajo. Se verificó que las patologías no estaban relacionadas con la excreción de calcio. Tampoco se encontró relación alguna entre la ingesta y la excreción de calcio. El estudio de la relación entre el sodio y la ingestión de proteínas con la excreción del calcio ha revelado una correlación positiva en ambos casos aunque débil. Se concluye, por tanto, que además de controlar la ingestión de calcio en los ancianos institucionalizados, es necesario ajustar las cantidades de sodio y proteínas para minimizar la pérdida de calcio en la orina.

Palabras clave: Ingestión, Excreción, Calcio, Ancianos, Bragança.

(t) 31 th October 2013

Accepted: 10th May 2015

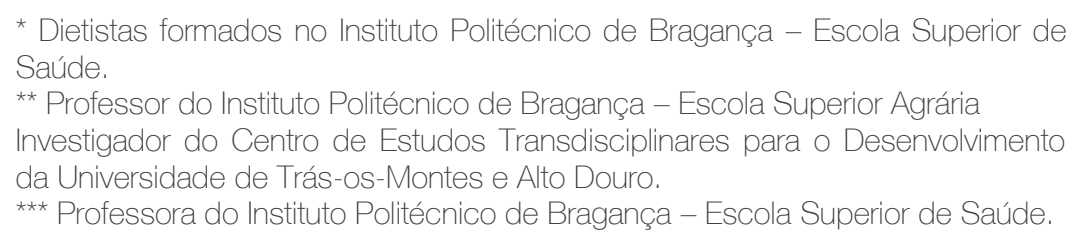




\section{Egitania \\ s c i e n c i a}

\section{INTRODUÇÃO}

Nas últimas décadas, tem-se assistido a um aumento da população idosa (com 65 ou mais anos). Efetivamente, em 1981, 11,4\% da população portuguesa estava incluída neste grupo etário. Em 2011, representava já cerca de 19\% da população (INE, 2011). Devido às inúmeras modificações biológicas provocadas pelo processo de envelhecimento, tais como alterações na ingestão alimentar, diminuição da área de superfície de absorção e de produção de suco gástrico tornam-se num grupo, especialmente, vulnerável (Mahan \& Escott-Stump, 2010; Ferry \& Alix, 2004; Kuczmarski \& Weddle, 2005). Por estas razões, a avaliação nutricional do idoso assume uma enorme importância. $\bigcirc$ estudo da população idosa institucionalizada tem particular importância pois, segundo Gazzotti, Albert, Pepinster e Petermans (2000), estes indivíduos tendem a encontrar-se em pior estado nutricional do que aqueles que vivem em casa.

A ingestão de cálcio, em particular, assume especial interesse, dado que é, amplamente, abordada no contexto da redução do risco de inúmeras patologias crónicas como a osteoporose e a hipertensão arterial, frequentes nas sociedades desenvolvidas (Boonen, Rizzoli, Meunier, Stone, Nuki \& Syversen, 2004; Griffith, Guyatt, Cook, Bucher \& Cook, 1999; Slattery, Neuhausen, Hoffman, Caan, Curtin, Ma et al., 2004). Contudo, a biodisponibilidade do cálcio, pode ser modificada por fatores endógenos como a idade e as condições fisiológicas, mas também por circunstâncias exógenas que interferem na sua absorção e excreção (Guéguen \& Pointillart, 2000).

Um importante fator que pode afetar a biodisponibilidade do cálcio é o sódio, uma vez que a ingestão elevada deste nutriente conduz a uma maior calciúria (Nieves, 2006). Este fator tem sido constatado em diversos estudo e populações, como em populações multi-étinicas (Blackwood, Sagnella, Cook \& Cappuccio, 2001), em adultos, tanto em homens quanto em mulheres, aumentando com a idade (Lim, 2011), em mulheres jovens (Bedford \& Barr, 2011), em mulheres pós menopásicas (Park, Joung, Cho, Sohn, Hur, Kim et al, 2015; Carbone, Bush \& Barro, 2003), sendo que este efeito (maior ingestão de sódio resulta em maior excreção de cálcio) é maior naquelas que têm menor ingestão de cálcio (Carbone, Bush \& Barro, 2003).

Este fato pode ser particularmente importante na saúde óssea, uma vez que pode reduzir a quantidade de cálcio disponível, reduzindo a densidade mineral óssea, que poderá conduzir a patologias ósseas, como a osteoporose. Os estudos mostraram que a excreção elevada de cálcio está relacionada com o aumento do marcador da reabsorção óssea em mulheres pósmenopáusicas (Park, Jee, Joung, Cho, Sohn, Jin et al, 2014), e com a redução da área de densidade mineral óssea da anca nas mulheres com menor ingestão de cálcio (Bedford \& Barr, 2011). No entanto, Carbone, Bush e Barro (2003) não encontraram efeito da excreção de sódio na densidade mineral óssea da anca. 
Outros estudos também indicam que o consumo excessivo de proteína estimula a excreção renal de cálcio (Fairweather-Tail \& Teucher, 2002; Cashman, 2002). Para além disso, um estudo encontrou uma correlação entre a excreção de cálcio e a ingestão de proteína animal em adultos, tanto em homens quanto em mulheres, tanto nos mais jovens quanto nos mais velhos. Porém, o mesmo estudo não encontrou uma correlação entre a excreção de cálcio e a ingestão de proteína vegetal (Itoh, Nishiyama \& Suyama, 1998). No entanto, este fator não deve afetar a saúde óssea, uma vez que um estudo recente aponta para um efeito positivo da ingestão de proteínas na saúde dos ossos, em condições de ingestão adequada de cálcio. Este mesmo estudo afirma, ainda, que a relação entre a proteína da dieta e risco de fratura ainda não é clara (Manganoa, Sahnia \& Kerstetterc, 2014).

O presente estudo pretende comparar o consumo de cálcio de origem animal com o de origem vegetal e a ingestão de proteína e sódio com os valores de referência; verificar se existe relação entre a ingestão dietética de cálcio, sódio e proteína com a excreção urinária de cálcio; bem como investigar se idosos com patologias apresentam diferenças na calciúria.

\section{METODOLOGIA}

Para atingir os objetivos propostos desenvolveu-se um estudo quantitativo, observacional, transversal e analítico que tem como núcleo de pesquisa a quantidade de cálcio consumido e excretado em idosos institucionalizados no concelho de Bragança ( $N=588)$.

Foram enviados pedidos de colaboração a 9 lares do concelho de Bragança, contudo apenas quatro $(N=162)$ responderam positivamente. Dos 162 idosos propostos foram excluídos 113 uma vez que 74 apresentavam limitações físicas (estavam acamados, em cadeira de rodas ou tinham mobilidade reduzida e usavam fralda no dia a dia ou apenas durante a noite), 51 apresentavam limitações psicológicas (confusão mental, incapacidade de assinar o consentimento informado de forma consciente). Por fim, foram excluídos 12 indivíduos por não estarem incluídos na definição de idosos (indivíduos com idade igual ou superior a 65 anos). Dos 49 idosos restantes, apenas 40 completaram, na totalidade, a recolha de dados.

A participação foi voluntária, sendo que cada participante foi informado relativamente aos objetivos e relevância do estudo, e também das garantias de anonimato e confidencialidade dos dados, respeitando a Declaração de Helsínquia (WHO, 2001). Foi obtido o consentimento informado, por escrito, por parte dos mesmos.

Para caracterizar a amostra foram recolhidos dados pessoais e antropométricos. Para avaliar o consumo de macro e micronutrientes foi realizado um recordatório de 24 horas e para a avaliação da calciúria foi recolhida a urina de 24 horas.

Nos dados pessoais, incluiu-se a idade, o género e a existência de patologias, designadamente, osteoporose, insuficiência renal, hipertiroidismo, hipotiroidismo, 


\section{Egitania}

s c i e $n$ c i a

hiperparatiroidismo, hipoparatiroidismo, raquitismo, osteomalacia, pancreatite, diabetes mellitus e hipertensão arterial.

$\mathrm{Na}$ avaliação antropométrica, os indivíduos foram pesados e medidos descalços e com roupas leves (balança SECA 869 e estadiómetro SECA 217). Procedeu-se ao cálculo e classificação do índice de massa corporal (IMC) em desnutrido (< 18,5 kg/m2), eutrófico (18,5 - 24,99 kg/m2), excesso de peso ( $\geq 25 \mathrm{~kg} / \mathrm{m} 2)$, obesidade grau I (30 - 34,99 kg/m2), obesidade grau II (35 - $39.99 \mathrm{~kg} / \mathrm{m} 2$ ) e obesidade grau III ( $\geq 40 \mathrm{~kg} / \mathrm{m} 2)$ segundo a classificação da WHO, 2000. Realizou-se a medição, através de um esfigmomanómetro OMRON, e classificação da tensão arterial em hipertenso $(\geq 140 \mathrm{mmHg}$ ou $\geq 90 \mathrm{mmHg}$ ) e não hipertenso ( $<90 \mathrm{mmHg}$ e $<140 \mathrm{mmHg}$ ). Efetuou-se a medição, através de uma fita métrica inextensível, e classificação da circunferência da cintura em risco elevado(> $102 \mathrm{~cm}$ para homens e $>88 \mathrm{~cm}$ para mulheres), risco moderado (>94 cm para homens e $>80 \mathrm{~cm}$ para mulheres) e baixo risco ( $<94 \mathrm{~cm}$ para os homens e $<80 \mathrm{~cm}$ para as mulheres) de doenças cardiovasculares e diabetes melitos (ESH/ESC, 2013).

O recordatório das 24 horas foi aplicado num único momento, fazendo-se referências às medidas caseiras para quantificar as porções dos alimentos consumidos. As medidas caseiras foram convertidas em gramas de alimentos cozinhados utilizando o Manual de quantificação de alimentos (Marques, Pinho \& Almeida, 1996) e, posteriormente, em valor nutricional utilizando a Tabela de composição de alimentos portuguesa (Instituto Nacional de Saúde Dr. Ricardo Jorge. 2006). Estimou-se a ingestão calórica total, de proteína, de sódio, de vitamina $D$, de cálcio total, de cálcio de origem animal, de cálcio de origem vegetal e de cálcio da água.

A recolha de urina de 24 horas foi efetuada para obter os valores de calciúria e realizada apenas uma vez.

A análise de dados foi efetuada através do programa SPSS (Statistical Package for Social Sciences), versão 21.0 para Microsoft Windows®. Para caraterizar a amostra foi realizado um estudo descritivo através do cálculo das frequências absolutas e relativas das variáveis categóricas. No caso das variáveis quantitativas, recorreu-se ao cálculo de medidas de tendência central (média (XT e mediana) e medidas de dispersão, nomeadamente, desviopadrão (DP), mínimo (Min.) e máximo (Max.) (Maroco, 2007; Pestana \& Gageiro, 2005).

Para realizar o estudo comparativo entre amostras independentes, foi utilizado, em alternativa ao teste T-Student (teste paramétrico), o teste Mann-Whitney-Wilcoxon (teste não paramétrico). Efetivamente, quando verificadas as condições de aplicação dos testes paramétricos, nomeadamente, a normalidade dos dados através do teste de KolmogoroSmirnov com a correção de Lilliefors ( $N \geq 30$ ) ou através do teste de Shapiro-Wilk ( $N<30)$; e, a homogeneidade das variâncias através do teste de Levene, pelo menos uma destas condições era violada (Maroco, 2007; Pestana \& Gageiro, 2005). 


\section{Egitania}

s c i e $n$ c i a

Para realizar o estudo comparativo entre amostras dependentes utilizou-se, em alternativa ao teste T-Student (teste paramétrico), o teste de Wilcoxon (testes não paramétrico). De fato, foi possível utilizar o teste de Wilcoxon pois a condição de aplicação exigida, designadamente, a distribuição das diferenças ser simétrica (Maroco, 2007; Pestana \& Gageiro, 2005) foi verificada. Efetivamente, quando calculado o coeficiente de assimetria, este situava-se entre 2 e 2 (Guimarães \& Cabral, 2007).

Para realizar o estudo correlacional utilizou-se o teste da correlação ordinal de Spearman (teste não paramétrico) em alternativa ao teste $R$ de Pearson (teste paramétrico) pois verificou-se que os dados não seguiam a Distribuição Normal (Maroco, 2007; Pestana \& Gageiro, 2005). Trata-se de um teste, em que o valor da estatística do teste é o coeficiente de correlação que pode variar entre -1 (correlação inversa perfeita) e 1 (correlação direta perfeita). Na correlação inversa perfeita, à medida que uma das variáveis em estudo aumenta, a outra diminui. Na correlação direta perfeita, à medida que uma das variáveis em estudo aumenta, a outra aumenta também (Maroco, 2007; Pestana \& Gageiro, 2005).

Os testes estatísticos referidos foram executados com um nível de confiança de 95\% a que corresponde um nível de significância ( $\alpha$ ) de 5\%. A decisão estatística foi tomada com base na probabilidade de significância ou valor de prova ( $p$-value).

\section{RESULTADOS}

Os idosos institucionalizados que participaram no estudo, tinham idades compreendidas entre os 67 e 96 anos, sendo a média de idades de 83,02 ( \pm 6,5) anos.

A maioria dos participantes era do género feminino (67,3\%); era não hipertensa $(57,1 \%)$; apresentava risco elevado de desenvolver doenças cardiovasculares e DM (79,6\%), avaliado através da circunferência abdominal; e, apresentava excesso de peso (38,8\%), avaliado através do IMC (tabela 1).

Tabela 1 - Caraterização dos participantes $(N=49)$

\begin{tabular}{|c|c|c|c|}
\hline \multirow{2}{*}{ Variável } & \multirow{2}{*}{ Categorias } & \multicolumn{2}{|c|}{ Frequências } \\
\cline { 3 - 4 } & & Absolutas (n) & Relativas (\%) \\
\hline \multirow{2}{*}{ Género } & Masculino & 16 & 32,7 \\
\hline \multirow{2}{*}{ Hipertensão } & Feminino & 33 & 67,3 \\
\cline { 2 - 4 } & Hipertenso & 21 & 42,9 \\
\cline { 2 - 4 } & Não hipertenso & 28 & 57,1 \\
\hline
\end{tabular}




\section{Egitania}

s c i e $\cap$ c i a

Tabela 1 - Caraterização dos participantes $(N=49)$ (continuação)

\begin{tabular}{|c|c|c|c|}
\hline \multirow{3}{*}{ Classificação da Circunferência da cintura } & Risco moderado & 5 & 10,2 \\
\cline { 2 - 4 } & Risco elevado & 39 & 79,6 \\
\cline { 2 - 4 } & Baixo risco & 5 & 10,2 \\
\hline \multirow{4}{*}{ Classificação do IMC } & Desnutrido & 1 & 2,0 \\
\cline { 2 - 4 } & Eutrófico & 4 & 8,2 \\
\cline { 2 - 4 } & Excesso de peso & 19 & 38,8 \\
\cline { 2 - 4 } & Obesidade grau I & 18 & 36,7 \\
\cline { 2 - 4 } & Obesidade grau II & 4 & 8,2 \\
\cline { 2 - 4 } & Obesidade grau III & 3 & 6,1 \\
\hline
\end{tabular}

A tabela 2 apresenta os resultados obtidos para a ingestão de macro e micronutrientes diária da população estudada destacando-se o cálcio total, cálcio de origem animal e vegetal, proteína e sódio. Verificou-se que o consumo de cálcio total ingerido variou de 178,4 mg a 1577,7 mg, enquanto o consumo de cálcio de origem animal e vegetal variou de 0 a 1238,6 mg e de 89,5 a 344,4 mg, respetivamente. O consumo de proteína variou de 13,3 g a 122,5 g enquanto o consumo de sódio variou de 1123,9 mg a 8472,3 mg.

Na comparação do cálcio consumido de origem animal com o cálcio consumido de origem vegetal, obteve-se um p-value de 0,000, através do teste de Wilcoxon. Como tal, ao nível de significância de 5\%, concluiu-se pela existência de diferenças estatisticamente significativas entre o consumo de cálcio de origem animal e vegetal. A mediana para o consumo do cálcio de origem animal foi de 651,6 mg, enquanto a mediana do cálcio de origem vegetal foi de $182,4 \mathrm{mg}$.

Tabela 2 - Caraterização da ingestão dietética e excreção de cálcio da amostra

\begin{tabular}{|c|c|}
\hline Variável & $\overline{\boldsymbol{X}} \pm$ DP (Min. - Máx.) \\
\hline Energia (Kcal/dia) & $1391,0 \pm 377,3(589,2-2273,2)$ \\
\hline Proteína $(\mathrm{g} / \mathrm{dia})$ & $73,261 \pm 25,67258(13,3-122,5)$ \\
\hline Vitamina D $(\mu \mathrm{g} / \mathrm{dia})$ & $1,8 \pm 1,6(0-10,3)$ \\
\hline Sódio $(\mathrm{mg} / \mathrm{dia})$ & $4152,8 \pm 1661,5(1123,9-8472,3)$ \\
\hline Cálcio total consumido $(\mathrm{mg} / \mathrm{dia})$ & $875,1 \pm 333,6(178,4-1577,7)$ \\
\hline Cálcio de origem animal $(\mathrm{mg} / \mathrm{dia})$ & $668,8 \pm 306,1(0-1238,6)$ \\
\hline Cálcio de origem vegetal $(\mathrm{mg} / \mathrm{dia})$ & $188,2 \pm 53,8(89,5-344,4)$ \\
\hline Cálcio da água $(\mathrm{mg} / \mathrm{dia})$ & $18,1 \pm 7,8(0,0-28,9)$ \\
\hline Cálcio excretado $(\mathrm{mg} / \mathrm{dia})$ & $50,5 \pm 88,3(10-580)$ \\
\hline
\end{tabular}

Como foi referido, para realizar o estudo da correlação da excreção de cálcio com o consumo de cálcio foi utilizado o teste de Spearman, obtendo-se um p-value = 0,394 superior ao nível 
de significância. Desta forma, pode afirmar-se que a excreção e a ingestão de cálcio não estão correlacionadas. Através dos p-values obtidos pelo teste de Spearman pode-se afirmar que a excreção urinária de cálcio depende do consumo de sódio (p-value = 0,040) e proteína (p-value $=0,045)$. Os coeficientes de correlação obtidos através do teste de Spearman representados na tabela 3 indicam que a correlação é direta, ou seja, à medida que aumenta a ingestão de sódio e proteína, aumenta a excreção de cálcio. No entanto, os coeficientes de correlação encontrados indicam que as correlações encontradas são fracas.

Tabela 3 - Correlação entre a ingestão de cálcio, sódio e proteína com a calciúria

\begin{tabular}{|c|c|c|c|}
\hline Variável 1 & Variável 2 & $\boldsymbol{\rho}$ de Spearman & P-value \\
\hline Cálcio total & Cálcio excretado & 0,138 & 0,394 \\
\hline Proteína & Cálcio excretado & 0,319 & $\mathbf{0 , 0 4 5}$ \\
\hline Sódio & Cálcio excretado & 0,326 & $\mathbf{0 , 0 4 0}$ \\
\hline
\end{tabular}

Conforme pode ver-se na tabela 4, os p-values obtidos pelo teste Mann-Whitney-Wilcoxon nas comparações da excreção de cálcio segundo as patologias (osteoporose, insuficiência renal, DM e HTA), são superiores ao nível de significância. Assim sendo, pode afirmar-se que a excreção de cálcio nos idosos estudados não é influenciada pelas patologias consideradas.

Tabela 4 - Comparação da excreção de cálcio segundo as patologias

\begin{tabular}{|c|c|c|c|c|c|}
\hline \multirow{2}{*}{ Patologias } & \multirow{2}{*}{ Categorias } & \multicolumn{2}{|c|}{ Frequências } & \multirow{2}{*}{ Mean Rank } & \multirow{2}{*}{ P-value } \\
\hline & & Absolutas (N) & Relativas (\%) & & \\
\hline \multirow{2}{*}{ Osteoporose } & Sim & 5 & 12,5 & 18,0 & \multirow{2}{*}{0,634} \\
\hline & Não & 35 & 87,5 & 20,9 & \\
\hline \multirow{2}{*}{ Insuficiência renal } & Sim & 7 & 17,5 & 13,7 & \multirow{2}{*}{0,095} \\
\hline & Não & 33 & 82,5 & 21,9 & \\
\hline \multirow{2}{*}{ Hipertensão arterial } & Sim & 17 & 42,5 & 19,8 & \multirow{2}{*}{0,685} \\
\hline & Não & 23 & 57,5 & 21,4 & \\
\hline \multirow{2}{*}{ Diabetes melitos } & Sim & 11 & 27,5 & 19,5 & \multirow{2}{*}{0,738} \\
\hline & Não & 29 & 72,5 & 20,9 & \\
\hline
\end{tabular}




\section{DISCUSSÃO}

Relativamente ao consumo de cálcio, a média foi de 875,1 \pm 333,6 mg, valores que estão de acordo com os do estudo realizado em mulheres com 70 ou mais anos de idade na região do Porto (Lucas, Costa \& Barros, 2005). Apesar dos valores serem consistentes com outros estudos, os idosos apresentam um consumo alimentar inadequado, insuficiente para atingir as recomendações de cálcio (Food and Nutrition Board, 2014). Isto é um facto preocupante, tendo em consideração que este mineral é imprescindível para a manutenção da saúde óssea entre outros mecanismos, especialmente, nesta faixa etária.

Na população portuguesa, a ingestão de cálcio de origem animal surge como a principal fonte deste mineral (Poínhos, Franchini, Afonso, Correia, Teixeira, Moreira et al., 2009), o que se encontra de acordo com os resultados obtidos nesta investigação.

Relativamente à ingestão de proteína e sódio, os valores obtidos revelaram estar acima dos valores recomendados (Food and Nutrition Board, 2014).Vários estudos sobre consumo alimentar confirmam a tendência de um alto consumo de proteínas e sódio na dieta por parte da população portuguesa (Poínhos, Franchini, Afonso, Correia, Teixeira, Moreira et al., 2009).

A média e desvio-padrão (50,5 $\pm 88,3 \mathrm{mg})$ relativos ao cálcio excretado encontram-se abaixo do expectável para a população estudada. Foley e Boccuzzi (2010) referem que, numa amostra de urina de 24 horas, seria de esperar 100 a 250 mg de cálcio. Contudo, o cálcio excretado é, extremamente, sensível a alterações na carga filtrada (MacFadyen, Nordin, Smith, Wayne \& Rae, 1965) o que pode ter interferido com os dados obtidos.

Breslau, Sakhaee e Pak (1985) encontraram uma correlação positiva ou direta entre a osteoporose e a excreção de cálcio. No entanto, a análise estatística efetuada não permitiu encontrar essa relação. Este resultado poderá estar relacionado com o facto do número de idosos com osteoporose ser baixo ( $N=5)$. Para além disso, a medição da excreção de cálcio com recurso a uma amostra de urina de 24 horas pode não ser suficiente para encontrar diferenças estatisticamente significativas na excreção de cálcio tendo em consideração esta patologia.

Os resultados deste estudo revelaram a inexistência de diferenças significativas entre os níveis de cálcio excretado pelos idosos com e sem HTA. Tais resultados são consistentes com os estudos de Taylor, Mount, Forman e Curhan (2006) e Martini, Cuppari, Cunha, Schor e Heilberg (1998) nos quais ficou demonstrado que os níveis de cálcio na urina não estão relacionados de forma sólida com a HTA.

O estudo de Raskin, Stevenson, Barilla \& Pak (1978) demonstrou que dez de vinte pacientes diabéticos não controlados tinham hipercalciúria $e$, destes, cinco foram submetidos a um tratamento com insulina o que permitiu que os valores de cálcio excretados na urina voltassem ao normal. Porém, o estudo de lain e Browna (1999) sugere que, após a administração de 


\section{Egitania \\ s c 1 e $n$ c i a}

insulina, os níveis de cálcio excretado aumentam. Neste estudo não foram encontradas diferenças estatisticamente significativas na excreção de cálcio quando a DM foi tida em consideração.

Alguns estudos indicam que a excreção urinária de cálcio diminui a partir dos estágios iniciais de insuficiência renal proporcionalmente à redução na taxa de filtração glomerular (Hsu, 1997; Popovtzer, Massry, Coburn \& Kleeman, 1969). Neste estudo, não foram encontradas diferenças estatisticamente significativas na excreção de cálcio tendo em consideração a insuficiência renal. Os resultados obtidos no presente estudo podem, eventualmente, derivar de um aumento ou diminuição da hormona da paratiroide que regula a absorção intestinal de cálcio (Hsu, 1997).

Verificou-se que não existe associação entre a excreção e a ingestão de cálcio nos idosos estudados $\bigcirc$ mesmo tinha já sido observado num estudo realizado em indivíduos saudáveis e em indivíduos com e sem urolitíase (Pearle, Calhoun \& Curhan, 2005). Embora os resultados possam refletir, em parte, a influência da vitamina D, a diferente quantidade de proteína ingerida, o fósforo e a não consideração de hábitos alimentares de vários dias. É, também, possível que os dados reforcem a não-linearidade da relação entre a ingestão de cálcio e o cálcio na urina. Dado que estudos anteriores relatam um "efeito plateau" para a ingestão de cálcio e de cálcio na urina, comparando apenas com valores extremos de ingestão de cálcio (Knapp, 1947; Taylor \& Curhan, 2009; Guimarães, Diniz, Guimarães, Cardoso, Souto \& Penido, 2002). Assim, a relação entre o cálcio ingerido e cálcio na urina em indivíduos com uma dieta livre não estão definidos, é necessário um maior número de dados para obter conclusões válidas e possíveis relações entre o nível de excreção de cálcio na urina e outros fatores, como a ingestão de cálcio e a idade, género e o peso corporal. Também o volume urinário pode ter interferido com os resultados, mas existem estudos que relatam a associação independente entre a excreção urinária de cálcio e o volume urinário (Lam, Aaplini \& Mitchell, 2000).

Estudos indicam que uma maior ingestão proteica é proporcional a maiores perdas de cálcio (Itoh, Nishiyama \& Suyama, 1998; FAOMHO, 2001). Através do teste de Spearman, também esta associação foi encontrada neste estudo. Efetivamente, descobriu-se uma correlação direta entre a excreção de cálcio e a ingestão estimada de proteína. Os resultados são consistentes com os obtidos em estudos levados a cabo em adultos, nos quais existiu o controlo da dieta, em que a proteína dietética foi manipulada, e a excreção de cálcio na urina foi medida. Apesar de vários modelos experimentais, e fontes de proteína diferentes, quase todos os estudos têm relatado uma relação positiva entre a ingestão de proteína e o cálcio urinário (Pannemans \& Schaafsma, 1997; Guimarães, Diniz, Guimarães, Cardoso, Souto \& Penido, 2002).

A ingestão de sódio tem sido conhecida por ser um importante preditor de excreção de cálcio (Devine, Criddle, Dick, Kerr \& Prince, 1995; FAOMHO, 2001; Cappuccio, Kalaitzidis, Duneclift \& Eastwood, 2000). Um estudo realizado em mulheres idosas caucasianas revelou que há 


\section{Egitania \\ s c i e $n$ c i a}

um efeito significativo da ingestão de sódio sobre a excreção de cálcio (Carbone, Bush, \& Barro, 2003). Como esperado, neste estudo foi encontrada uma relação entre ingestão de sódio e a excreção de cálcio, indicando que uma maior ingestão de sódio corresponde a uma maior excreção urinária de cálcio. No entanto, a ingestão de sódio estimada na dieta a partir de registos dietéticos pode não ser, totalmente, confiável devido à variabilidade no teor de sódio de uma preparação para outra da mesma alimentação e, também, por causa da dificuldade em avaliar o uso do sal adicionado.

\section{CONCLUSÕES}

A população estudada apresenta consumo inadequado de vários nutrientes, nomeadamente, cálcio, sódio e proteína, podendo resultar em riscos para a saúde. Apesar de não ter sido encontrada a correlação entre o cálcio consumido e o excretado pela urina foram identificados fatores que interferiram na excreção de cálcio. A ingestão de proteína e de sódio foram correlacionados, positivamente, com a excreção de cálcio. Assim, para além de controlar a ingestão de cálcio em idosos institucionalizados, é necessário adequar os valores de sódio e proteína para os recomendados pelo Food and Nutrition Board (2014) e, desta forma, minimizar as perdas de cálcio na urina.

Na população idosa constata-se uma nítida e progressiva deterioração do estado nutricional. Tal deterioração ficou, claramente, evidenciada neste e noutros estudos. Assim, torna-se necessário um acompanhamento dos idosos institucionalizados por parte de profissionais capacitados, nomeadamente, dietistas e nutricionistas.

Devido aos condicionantes que a faixa etária investigada padece, o número de indivíduos envolvidos foi reduzido o que poderá estar na origem da ausência de diferenças significativas nos resultados, pelo que não se podem extrapolar os resultados obtidos para o universo de idosos institucionalizados.

\section{REFERÊNCIAS BIBLIOGRAFICAS}

BedFord, J.L. \& BarR, S.I. (2011). Higher Urinary Sodium, a Proxy for Intake, Is Associated With InCReAsed Calcium Excretion and lower Hip Bone Density in Healthy Young Women with Lower Calcium Intakes. Nutrients, 3, 951-61.

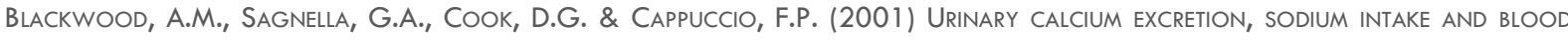
pressure in a multi-ethnic population: results of the Wandsworth Heart and Stroke Study. Journal of Human Hypertension, 15, 229-37.

Boonen, S., Rizzol, R., Meunier, P., Stone, M., Nuki, G., \& Syversen, U. (2004). The need for Clinical guidance in the use Of CalCium AND VITAMIN D IN THE MANAGEMENT OF OSTEOPOROSIS: A CONSENSUS REPORT. OSTEOPOROSIS INTERNATIONAL, 5, 511 -9. 


\section{Egitania \\ s c i e $n$ c i a}

Breslau, N., SakHaee, K., \& Pak, C. (1985). Impaired adaptation to Salt-induced urinary Calcium losses in postmenopausal osteoporosis. Transactions of the Association of American Physicians, 98, 107-15.

Cashman, K.D. (2002). Calcium Intake, CAlCiUm biOavailability AND bONe health. British Journal Of Nutrition, 87, S169-77.

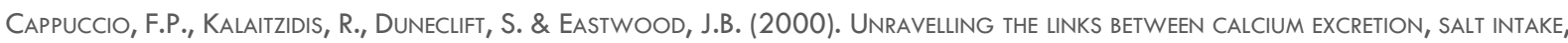
HYPERTENSION, KIDNEY STONES AND BONE METABOLISM. JOURNAL OF NEPHROLOGY, 13, 169-77.

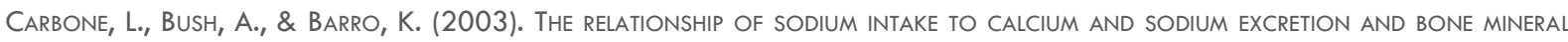
density of the hip In postmenopausal. Jounal of Bone and Mineral Metabolism, 21, 415-20.

Devine, A., Criddle, A., Dick, M., Kerr, A., \& Prince, L. (1995). A longitudinal Study of the efFect of SOdium and Calcium intakes ON regional bone density in postmenopausal women. The American Journal of Clinical Nutrition, 62, $740-5$.

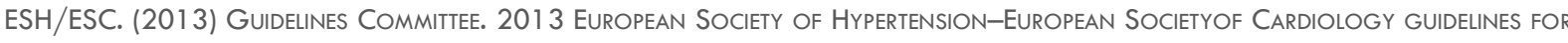
THE MANAGEMENT OF ARTERIAL HYPERTENSION. JOURNAL OF HYPERTENSION, 31, 1 281-357.

faO/WhO. (2001). In Human Vitamin and Mineral Requirements. Report of a Joint Food agriculture Organization/World Health Organization Expert Consultation.

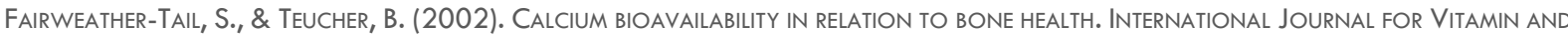
NUTRITION RESEARCH, 72, 12-18.

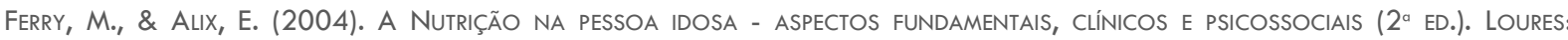
LUSOCIÊNCIA.

Foley, K., \& BoccuzzI, L. (2010). Urine Calcium: Laboratory Measurement and Clinical Utility. Laboratory Medicine, 41, 683-6.

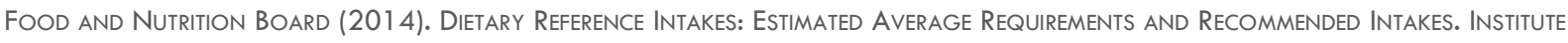
OF MEDICINE. ACEDIDO EM 21 DE ABRIL DE 2015. DISPONIVEL EM: WWW.NAL.USDA.GOV/FNIC/DRI/DRI_TABLES/RECOMMENDED_INTAKES_INDIVIDUALS.PDF

Gazzotti, C., Albert, A., Pepinster, A., \& Petermans, J. (2000). Clinical usefulness of the Mini Nutritional Assessment (MNA) scale in Geriatric medicine. The Journal of NUtrition, Health \& AGING, 4, 176-81.

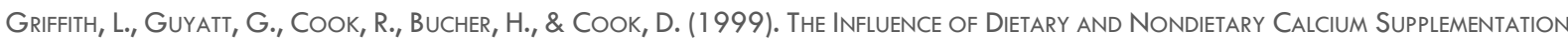
on Blood Pressure - AN Updated Meta-Analysis of Randomized Controlled Trials. American Journal of Hypertension, $12,84-92$.

Guimarães, M., Diniz, J., Guimarães, M., Cardoso, R., Souto, M., \& Penido, M. (2002). ExCreção urinária de cálcio, ÁCido úrIco e citrato. Jornal de Pediatria da Sociedade Brasileira de Pediatria, 78, 153-60.

Guimarães, R. \& CABral, J. (2007). Estatística. LisboA: MCGraW-Hill.

Guéguen, l., \& Pointillart, A. (2000). The Bioavallability of Dietary Calcium. Journal of the American College Of Nutrition, 19, S119-36.

Hsu, H. (1997). Are We mismanaging Calcium and phosphate metabolism in renal fallure? American Journal Of Kidney Diseases, 29, 641-9.

IAIN R. \& BROWNA, A. (1999). SEX DIFFERENCE IN THE RELATIONSHIP OF CALCIUM AND MAGNESIUM EXCRETION TO GLYCAEMIC CONTROL IN TYPE 1 diabetes mellitus. Clinica Chimica ACta, 283, $119-28$.

INE (2011). Censos 2011 - Resultados Provisórios. Lisboa: Instituto Nacional de Estatística.

instituto Nacional de Saúde Dr. Ricardo Jorge (2006). TAbela da Composição dos Alimentos. Lisboa: Editorial do Ministério da EDUCAÇÃO.

ITOH, R., NISHIYAMA, N., \& SUYAMA, Y. (1998). DIETARY PROTEIN INTAKE AND URINARY EXCRETION OF CALCIUM: A CROSS SECTIONAL STUDY IN A heAlthy JaPANESE POPULATION. AMERICAN JOURNAL OF CliniCAL NUTRITION, 67, 438-44. 


\section{Egitania \\ s c i e $\cap$ c i a}

Lim, H. (2011). A Study on the Calcium and SOdium Intakes and Urinary Calcium Excretion of Adults in Busan. Korean Journal Of COMMUNITY NUTRITION, 16, 215-226.

KNAPP, E. (1947). FACTORS INFLUENCING the URINARY EXCRETION OF CALCIUM IN NORMAL PERSONS. The JOURNAL OF CLINICAL INVESTIGATION, $26,182-202$.

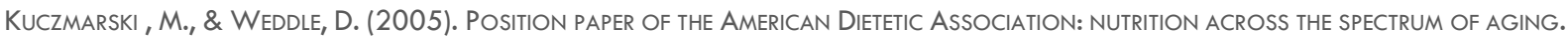
JOURNAL OF AMERICAN Dietetic ASSOCIATION, 105, 616-33.

Lam, G., Aaplini, J., \& Mitchell, L. (2000). Does a high concentration of Calcium in the urine cause an important renal CONCENTRATING DEFECT IN HUMAN SUBJECTS? CLINICAL SCIENCE, 98, $313-9$.

lucas, R., Costa, L., \& Barros, H. (2005). ingestão de Cálcio e Vitamina D numa amostra Urbana de Mulheres Portuguesas. ARQUIVOS DE MEDICINA, 19,7-14.

MacFadyen, J., Nordin, C., Smith, A., Wayne, J., \& Rae, L. (1965). Effect of Variation in Dietary Calcium on Plasma Concentration

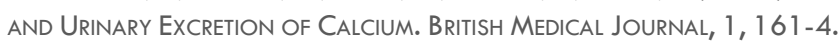

MAhan, K., \& Escott-Stump, S. (2010). Krause - Alimentação, Nutrição e Dietoterapia (1 $2^{\circ}$ ed.). Rio de Janeiro: ElseVier Editora.

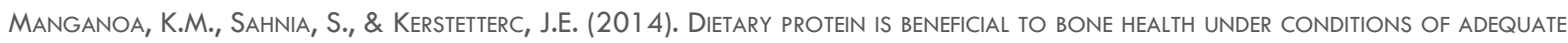
Calcium intake: an update On Clinical research. CURrent Opinion in Clinical Nutrition \& Metabolic Care, 17, 69-74.

MAROCO, J. (2007). ANÁlISE EstatístiCA COM UtILIZAÇÃo do SPSS. LISBOA: EdIçÕES SílAbO.

Marques, M., Pinho, O., \& Almeida, MDV. (1996). Manual de quantificação de alimentos (1ª Edição ed.). Porto: Faculdade de CIÊNCIAS da NUtriç̧ão e AlimentaÇão da UniVersidade do Porto.

Martini, A., Cuppari, L., Cunha, A., Schor, N., \& Hellberg, P. (1998). Potassium and sodium intake and exCretion in CalCium Stone FORMING PATIENTS. JOURNAL OF RENAL NUTRITION, 8, $127-31$.

Nieves, J. (2006). Osteoporosis: the role Of micronutrients. The American Journal of CliniCal Nutrition, 81, S1 232-9.

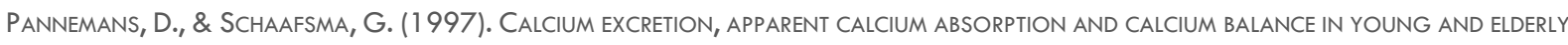
SUBJECTS: INFLUENCE OF PROTEIN INTAKE. BRITISH JOURNAL OF NUTRITION, 77, 72 1-9.

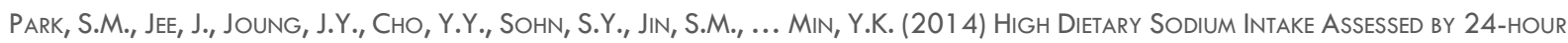

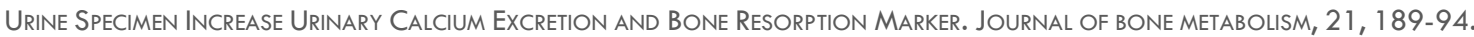

Park, S.M., Joung, J.Y., Cho, Y.Y., SOHN, S.Y., HUR, K.Y., KIM, J.H., ... MIN, YK. (2015) EFFeCt OF high dietARY SODIUM ON bONE TURNOVER MARKERS AND URINARY CALCIUM EXCRETION IN KOREAN POSTMENOPAUSAL WOMEN WITH LOW BONE MASS. EUROPEAN JOURNAL OF CLINICAL NUTRITION, 69, 361-6.

Pearle, M., Calhoun, E., \& Curhan, G. (2005). Is 24-hors urinary Calcium a surrogate marker? The Journal of Urology, 3, 45962.

Pestana. M. \& Gageiro, J. (2005). AnÁlise de Dados para CiênCias Sociais: A complementaridade do SPSS. Lisboa: Edições Sílabo.

Poínhos, R., Franchini , B., Afonso , C., Correia, F., Teixeira, V., Moreira , P., ... Almeida, M.D.V. (2009). AlimentaÇão e estilos de

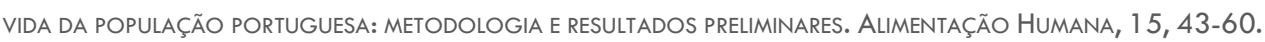

Popovtzer, M., Massry, G., Coburn, W., \& Kleeman, R. (1969.). The interrelationship between sodium, Calcium, and magnesium eXCretion in adVANCed renal fallure. Journal of Laboratory and Clinical Medicine, 73, 763-71.

Raskin, P., Stevenson, R., Barilla, E., \& PaK, Y. (1978). The hypercalciuria of diabetes mellitus: its amelioration With insulin. Clinical ENDOCRINOLOGY, 9, 329-35.

Slattery, M., Neuhausen, S., Hoffman, M., CaAn, B., Curtin, K., Ma, K. \& Samowitz, W. (2004). Dietary calcium, vitamin D, VDR GENOTYPES AND COLORECTAL CANCER. INTERNATIONAL JOURNAL OF CANCER, $111,750-6$. 


\section{Egitania \\ s c i e $n$ c i a}

Taylor, E., \& Curhan, G. (2009). Demographic, Dietary, and Urinary factors and 24-h Urinary Calcium Excretion. Clinical Journal OF THE AMERICAN SOCIETY OF NePHROLOGY, 4, 1980-7.

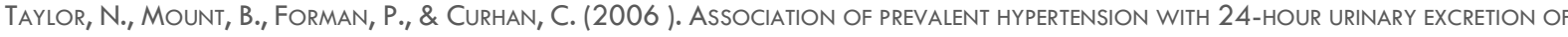
CALCIUM, CITRATE, AND Other FACTORS. AMERICAN JOURNAL OF KIDNEY DISEASES, 47, 780-9.

WHO (2000). Obesity: preventing and managing the global epidemic. Report of a WHO Consultation. WHO technical Report Series 894. Geneva: World Health Organization.

WhO (2001). Declaration of Helsinki - Ethical Principles for Medical Research Involving Human Subjects. Bulletin Of the World Health Organization, 79. 